\title{
A Learning Based Model for Headline Extraction of News Articles to Find Explanatory Sentences for Events
}

\author{
Sandip Debnath ${ }^{1,3}$ \\ Department of Computer Sciences and \\ Engineering ${ }^{1}$ \\ Penn State University \\ University Park, PA 16802, USA \\ debnath@acm.org
}

\author{
C. Lee Giles ${ }^{1,2,3}$ \\ School of Information Sciences and Technology 2 \\ eBusiness Research Center ${ }^{3}$ \\ Penn State University \\ University Park, PA 16802, USA \\ giles@ist.psu.edu
}

\begin{abstract}
Metadata information plays a crucial role in augmenting document organising efficiency and archivability. News metadata includes DateLine, ByLine, HeadLine and many oth$\mathrm{ers}^{1}$. We found that HeadLine information is useful for guessing the theme of the news article. Particularly for financial news articles, we found that HeadLine can thus be specially helpful to locate explanatory sentences for any major events such as significant changes in stock prices. In this paper we explore a support vector based learning approach to automatically extract the HeadLine metadata. We find that the classification accuracy of finding the HeadLines improves if DateLines are identified first. We then used the extracted HeadLines to initiate a pattern matching of keywords to find the sentences responsible for story theme. Using this theme and a simple language model it is possible to locate any explanatory sentences for any significant price change.
\end{abstract}

\section{Categories and Subject Descriptors}

H.4 [Information Systems Applications]: Miscellaneous; I.7 [Document and Text Processing]: Electronic Publishing; I.7 [Document and Text Processing]: Miscellaneous

\section{Keywords}

Metadata Extraction, News Metadata, Explanatory Sentence

\section{General Terms}

Algorithms, Experimentation

\footnotetext{
${ }^{1}$ Please see NewsML 1.2 DTD specifi cation for more details
}

Copyright is held by the author/owner(s).

$K$-CAP'05, October 2-5, 2005, Banff, Alberta, Canada ACM 1-59593-163-5/05/0010 .

\section{HEADLINE EXTRACTION}

In our earlier paper [2], we described in detail, how to use a support vector based learning approach to accurately identify the DateLines of news articles. In short we first devised our own temporal grammar by which we locate all the temporal expressions in an article. Next we create a vector of properties for each of these expressions to train a support vector classifier. Using this classifier we can achieve high accuracy in finding the Base Time-line (BT) or DateLine. Here we show that using the same approach, we can reach higher accuracy of finding the HeadLine it we do it step-by-step. It means that the accuracy of finding the HeadLine increases if we first identify the DateLine location and use this as part of the feature vector to train the HeadLine classifier than train the classifier without the DateLine information. The classification accuracy of finding the DateLine is shown in [2] and so is not shown here. The classification accuracy of HeadLine is shown in table 1.

\section{FINDING STORY THEME}

To the best of our knowledge, finding the theme of a news article based on its HeadLine has not been explored yet. News articles consist of one or more paragraphs devoted to the main story which the HeadLine refers. After extracting the HeadLine, we used Brill's POS tagger [1] to tag each word. Prepositions, conjunctions, interjections, and other low entropy words such as "a", "and", "the" etc. are removed from the word-set of HeadLine. Next we use Krovetz [3] stemming to get the roots of these filtered words. We then create a language model $M_{h}$ for the HeadLine. It represents a discrete distribution over the words in the vocabulary. We assume that the word distribution inside paragraph $P_{i}$ in a story is independent of of the distributions of other words. Assuming this and assuming that the distribution of each paragraph generation is also independent, we analyse the paragraphs in the same way as the HeadLine. Now we can estimate the maximum likelihood of a paragraph $P_{i}$ so that it would be generated by the model $M_{h}$ (for the HeadLine h). The sentences of this "theme" paragraph are taken as the theme of the story and to find explanatory sentences. Due to the space constraints, we can not show the details of the result, 


\begin{tabular}{|l|l|l|l|}
\hline Site & $\begin{array}{l}\text { Number } \\
\text { of articles }\end{array}$ & $\begin{array}{l}\text { Accuracy } \\
\text { (w/o) of } \\
\text { HeadLine }\end{array}$ & $\begin{array}{l}\text { Accuracy } \\
\text { (w) of } \\
\text { HeadLine }\end{array}$ \\
\hline \hline AP & 37 & 82.35 & 94.11 \\
\hline Briefing.com & 132 & 87.12 & 90.9 \\
\hline BusinessWeek & 128 & 88.28 & 93.75 \\
\hline Business Wire & 430 & 84.65 & 92.3 \\
\hline CBS MarketWatch & 606 & 90.26 & 94.88 \\
\hline CCBN & 92 & 80.43 & 85.86 \\
\hline Dow Jones & 324 & 91.97 & 95.67 \\
\hline EDGAR Online & 599 & 90.65 & 96.82 \\
\hline Forbes & 451 & 88.91 & 95.34 \\
\hline Market Wire & 158 & 89.87 & 94.93 \\
\hline Morningstar & 14 & 71.42 & 71.42 \\
\hline Motley Fool & 162 & 82.71 & 89.51 \\
\hline NewsFactor & 71 & 91.5 & 95.77 \\
\hline PR Newswire & 525 & 94.66 & 96.19 \\
\hline PrimeZone & 64 & 85.93 & 90.62 \\
\hline Reuters & 641 & 93.6 & 96.72 \\
\hline SmartMoney & 63 & 87.3 & 92.06 \\
\hline StarMine & 38 & 89.47 & 84.21 \\
\hline TheStreet.com & 351 & 91.16 & 92.59 \\
\hline \hline
\end{tabular}

Table 1: The columns represent Source Web-site, Total Number of Articles, Accuracy of HeadLine without the use of DateLine information and Accuracy of HeadLine with the use of DateLine information as an extra parameter.

however we can just mention that for a limited number of news stories we achieved over $97 \%$ of paragraph-based FMeasure.

\section{EXPLANATORY SENTENCES}

Explaining a stock price change for a company is a complex and hard problem. To the best of our knowledge, it has not received enough attention. Our work [4] on finding "keyword-based" explanation uses word entropy measures for news articles during the price change $\left(W_{A}\right)$ compared to the past or historical set $\left(W_{B}\right)$ of news articles as shown in figure 1. For artificial market events, it worked pretty well.

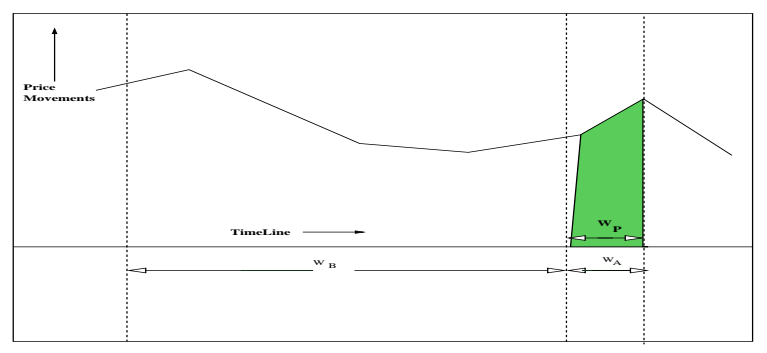

Figure 1: A sample price change curve. $W_{A}$ and $W_{B}$ are the time-line windows for considering the document collections to compare (in our previous work[4]) for possible explanatory keywords. Here we consider $W_{P}$ as the time window to find the possible explanatory sentence $(s)$.

\begin{tabular}{|l|l|l|}
\hline Company & Date & Sentence \\
\hline \hline MSFT & $\begin{array}{l}\text { July 1, 1, } \\
2005\end{array}$ & $\begin{array}{l}\text { IBMCorp. will get \$775 million in cash } \\
\text { and \$75 million worth of software from } \\
\text { Microsoft Corp. to settle claims ... }\end{array}$ \\
\hline C & $\begin{array}{l}\text { July 11, } \\
2005\end{array}$ & $\begin{array}{l}\text { Merrill Lynch cut its second-quarter } \\
\text { earnings estimate for Citigroup, Inc. to } \\
\text { \$1.04 from \$1.09 a share and slashed its } \\
\text { fi scal 2005 earnings estimate... }\end{array}$ \\
\hline \hline
\end{tabular}

Table 2: Explanatory sentences for companies for a specified date

We wanted to extend the same idea to generate explanatory sentences for any significant price change. However the entropy based model does not work well for sentence generation. We slightly borrowed the concept from Ponte et. al [5] for the use of language models representing the trends. We used the models $M_{p t}$ of price trends for sentences in the "theme" paragraphs of the articles. These articles are selected for the particular price change according to temporal relationship $\left(W_{P}\right)$. That means that the sentence in the following equation is taken as the explanatory sentence:

$$
S_{\text {expl }}=\operatorname{argmax}_{j \in \sum_{i}^{Q}\left|S_{i}\right|} \operatorname{Prob}\left(S_{j} \mid M_{p t}, \sum_{q}^{Q} P_{\text {winner }}^{q}\right)
$$

where there are total $Q$ stories selected for the price change, and each has one "theme" paragraph and the total number of sentences in all these paragraphs is $\sum_{i}^{Q}\left|S_{i}\right|$. Due to space constraints, we can not show full results; table 2 shows results for two recent price changes of two companies.

\section{CONCLUSION}

We devised a novel approach to extract news HeadLines using SVM and using them to find story themes to get a sentence based explanation for a stock price change.

\section{REFERENCES}

[1] E. Brill. A simple rule-based part of speech tagger. In proceedings of ANLP, pages 152-155, 1992.

[2] S. Debnath, P. Mitra, and C. L. Giles. Finding base time-line of a news article. In proceedings of FLAIRS, pages 142-147, 2005.

[3] R. Krovetz. Viewing morphology as an inference process. In Artif. Intell., volume 118(1-2), pages 277-294, 2000.

[4] D. M. Pennock, S. Debnath, E. J. Glover, and C. L. Giles. Modelling information incorporation in markets, with application to detecting and explaining events. In proceedings of UAI, pages 405-413, 2002.

[5] J. M. Ponte and W. B. Croft. A language modeling approach to information retrieval. In Research and Development in Information Retrieval, pages 275-281, 1998. 